\title{
Diversity Analysis of Non-Coherent Wireless Network Coding
}

\author{
Wei Guan and K. J. Ray Liu
}

\begin{abstract}
Network coding has been widely used in the wireless uplink. To do coherent detection at the receiver, channel estimation is performed to collect the channel state information (CSI). For multi-user channels, the channel estimation overhead is sometimes formidable and may even outweigh the network coding gain. To address this issue, we study the non-coherent network coding in this work. Both the analog network coding (ANC) and digital network coding (DNC) are studied. Depending on the available CSI, we first develop the coherent and non-coherent maximum likelihood receivers. For ANC, as the non-coherent receivers have no closed form, we develop two suboptimum receivers according to the average link qualities. Next we study the error rates, and show that full diversity can always be achieved at extremely high signal-to-noise ratios (SNR) regardless of the CSI assumptions. However, the lack of perfect CSI would incur some diversity loss at modest SNRs. Besides, the performance loss of ANC is more serious due to the incapability to efficiently suppress multi-user interferences at the receivers. Extensive simulations are performed to verify our analytical results.
\end{abstract}

Index Terms-Network coding, non-coherent modulations, diversity.

\section{INTRODUCTION}

$\mathbf{U}$ SER cooperation is a new communication paradigm in which some relay nodes are selected to help forward the source messages in order to provide spatial diversity, extend transmission coverage and save transmitted power [1]. Some early work in this area [2] has studied several repetition-coding based cooperation protocols. However, these schemes are not bandwidth efficient due to the half-duplex constraints of the wireless devices. This is because in practice, the relay nodes generally cannot transmit and receive on the same channel at the same time, thus two orthogonal channels are required to deliver a single source message and the bandwidth efficiency is lowered by one half.

When the relay nodes have to serve multiple users, such rate loss can be mitigated to some extent by leveraging network coding [3]. The idea of network coding is to mix different source messages at the relay nodes to form a single networkcoded message, based on which all the end nodes may still be able to extract the desired information. In practice, the source data can be combined in the finite field through digital network coding (DNC) [4]; however, as the relay nodes may decode the wrong messages, the decoding errors would propagate to

Manuscript received June 7, 2012; revised October 15, 2012; accepted January 7, 2013. The associate editor coordinating the review of this paper and approving it for publication was A. Ghrayeb.

The authors are with the Department of Electrical and Computer Engineering, University of Maryland, College Park, MD 20742 (e-mail: \{wguan, kjrliu\}@umd.edu).

Digital Object Identifier 10.1109/TWC.2013.022113.120814 the intended receivers and lower the diversity performances [5]. Alternatively, the source data can also be combined in the complex field through analog network coding (ANC) [6], in which the intermediate nodes no longer need to decode the source messages. For both strategies, channel state information (CSI) is very important for the receivers to mitigate error propagation [7] or suppress multi-user interferences (MUI) $[8,9]$.

However, perfect CSI is not always available in practice. This could occur when the channels experience fast fading such that it is really hard to track the real-time channel variations at the receiver. Channel estimation overhead is another concern. For the multi-user relay channel, the channel estimation overhead increases linearly with the product of the number of users and the number of relays, which may become formidable and even outweigh the network coding gain. Besides, channel estimation requires additional wireless resources such as power and dedicated channels that could be otherwise used to transmit the data stream, so the bandwidth efficiency is also reduced to some extent. Because of these concerns, non-coherent modulations such as differential modulations and orthogonal signalling (e.g., ON-OFF keying (OOK) and frequency shift keying (FSK)) that do not require perfect CSI are of more practical interests under these circumstances.

Non-coherent modulations have been widely studied in the context of single-source uncoded relaying networks. For analog relaying, the maximum likelihood (ML) receiver for FSK modulations is developed in [10]. Although the ML receiver generally has no closed form, the upper and lower bounds on the average bit error rate (BER) are obtained in [11], and it is observed that full diversity can only be achieved for FSK modulations but not for OOK schemes. In [12], a near-ML receiver is developed and simulation results show that this scheme can also achieve full diversity. Two blind detection schemes are proposed in [13] and [14] based on maximum energy selection and generalized likelihood ratio test, respectively. The advantage of these blind detectors is that the receivers do not need to know the statistical information of the channels, which further reduces the signalling overhead. For digital relaying, [15] obtains the error rates of FSK modulations by developing a piecewise linear approximation for the ML decision metrics. A threshold-based selection relaying protocol is developed in [16] and the corresponding BER is analyzed. For multi-relay networks, [17] proposes a relay selection method and shows that full diversity can be achieved.

Some literatures have also studied non-coherent modula- 
tions for the two-way relay channel using network coding. In [18], a set of differential detectors are developed, and the BER performance is studied in [5]. A relay selection strategy that can achieve full diversity is developed in [19], where the end nodes use differential modulations. For OOK modulations, a simple threshold-based energy detection scheme is developed in [20], and the optimum threshold is obtained in closed form. In [21], the non-coherent relay receiver is developed for binary FSK modulations, where physical-layer DNC is used at the relay nodes. The optimum and suboptimum receivers are developed in [22] and the bounds on BER are also obtained.

In practice, network coding has also been widely applied in the wireless uplink, but very few literatures have discussed using non-coherent modulations for this common application. Different from two-way relay channel, where the end nodes may eliminate its self-interferences and the bidirectional communication can be decomposed into two parallel one-way relaying channels, the wireless uplink channel is much more analytically challenging as the receiver has to jointly decode all the source signals, potentially in the presence of MUI $[8,9]$. Besides, the wireless uplink may possibly accommodate multiple users and thus is more sensitive to the channel estimation overheads. So to provide a comprehensive study on this important application is very meaningful, and such concerns motivate the current work.

To be specific, we study the two-user wireless uplink channels using FSK modulations. Both ANC and DNC are studied, where the source signals are combined in the complex field and finite field, respectively. We first design the coherent and non-coherent ML receivers when global CSI and statistical CSI is available at the receivers, respectively. For ANC, as the non-coherent $\mathrm{ML}$ receiver has an intractable integral form, we develop two suboptimum receivers that are near optimum depending on the relative quality of source-relay channel and relay-destination channel. The pairwise error probability (PEP) is then studied, and the scaling laws of different PEPs are derived at high signal-to-noise ratios (SNR). It is demonstrated that full diversity is always achievable regardless of the CSI assumptions; however, using non-coherent detection would incur some diversity loss at modest SNRs such that the resulting error rates do not decrease that fast compared to coherent detection. Besides, we show that the performance loss of ANC is more serious due to the incapability to efficiently suppress MUI at the receiver.

The rest of this paper is organized as follows: We describe the system model in section II, and then present transceiver design in section III. The error rates are studied in section IV, and some simulation results are given in section V. Finally some conclusions are drawn in section VI.

Notations: $|\cdot|,(\cdot)^{T}$ and $(\cdot)^{H}$ stand for absolute value, transpose and conjugate transpose, respectively. The boldface lowercase letter $\mathbf{a}$ and the boldface uppercase letter $\mathbf{A}$ represent vector in column form and matrix, respectively. $\|\mathbf{a}\|$ and $\operatorname{det} \mathbf{A}$ denote the Euclidean norm of a vector a and the determinant of a square matrix $\mathbf{A}$, respectively. We shall use abbreviation i.i.d. for independent and identically distributed. We denote $Z \sim \mathcal{C N}(\mathbf{u}, \mathbf{\Sigma})$ as a circularly symmetric complex Gaussian random variable vector with mean $\mathbf{u}$ and covariance matrix $\boldsymbol{\Sigma}$, $Z \sim \exp (\mu)$ as an exponential random variable with mean $\mu$,

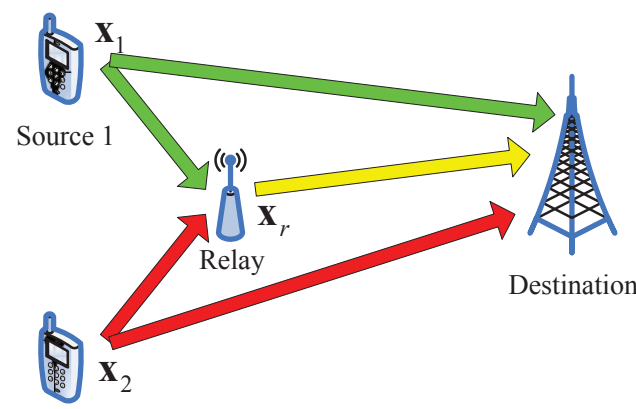

Source 2

$3^{\text {rd }}$ phase

Fig. 1. System model of the two-user network-coded uplink.

and $Z \sim \chi_{k}^{2}$ as a chi-square random variable with the degree of freedom being $k$. The probability of an event $\mathcal{A}$ is denoted by $\operatorname{Pr}(\mathcal{A})$. The cumulative distribution function (CDF) and the probability density function (PDF) of a random variable $Z$ are denoted by $F_{Z}(z)$ and $f_{Z}(z)$, respectively. Finally, we say $h(x)=\mathrm{O}(g(x))$ if $\limsup _{x \rightarrow \infty} \frac{h(x)}{g(x)}<\infty$.

\section{SySTEM MODEL}

Consider the wireless uplink channels with two source nodes sending data to a common destination with the help of a single relay node, as shown in Fig. 1 . Let $f_{k} \sim \mathcal{C N}(0,1)$ and $h_{k} \sim \mathcal{C N}(0,1)$ for $k=1,2$ be the channel coefficients from the $k$ th source to the relay and to the destination, respectively, and $g \sim \mathcal{C N}(0,1)$ be the channel coefficient from the relay to the destination. All the channel coefficients are independent, and the additive noises on different channels are also i.i.d. $\mathcal{C N}(0,1)$. The channel gains are denoted by $\lambda_{s r}, \lambda_{s d}$ and $\lambda_{r d}$ for source-relay channels, source-destination channels and relay-destination channel, respectively. The channel gains are some constants that are determined by the distances and the path-loss exponents.

Only uncoded systems are considered throughout this work, i.e., there is no error detection/correction code. The two source nodes are supposed to use the M-ary FSK modulations. The symbol set is denoted by $\Omega=\left\{\mathbf{e}_{1}, \mathbf{e}_{2}, \cdots, \mathbf{e}_{M}\right\}$, where $\mathbf{e}_{l}$ is the $l$ th unit vector with the $l$ th element being 1 and the other elements being 0 . The whole data transmission is completed in three phases. In the $k$ th phase for $k=1,2$, the $k$ th source node broadcasts its own signals while the other source node keeps silent. The received signals at the relay node and at the destination are denoted by

$$
\begin{aligned}
& \mathbf{y}_{k r}=\sqrt{P \lambda_{s r}} f_{k} \mathbf{x}_{k}+\mathbf{n}_{k r}, \\
& \mathbf{y}_{k d}=\sqrt{P \lambda_{s d}} h_{k} \mathbf{x}_{k}+\mathbf{n}_{k d},
\end{aligned}
$$

respectively. Here $P$ is the transmitted power, $\mathbf{x}_{k}$ is the $k$ th source symbol with $\mathbf{x}_{k} \in \Omega, \mathbf{n}_{k r}$ and $\mathbf{n}_{k d}$ are the corresponding additive noises. As the noise power has been normalized, the transmitted power $P$ is also treated as the system SNR throughout this work. The relay operation is dependent upon the network coding schemes that would be detailed later. At this moment, we simply denote the relay symbol by $\mathbf{x}_{r}$ and assume it has unit power, i.e., $E\left\|\mathbf{x}_{r}\right\|^{2}=1$. Then in the third phase, the relay node forwards its symbol to 


$$
\begin{aligned}
& L\left(\mathbf{y}_{r d} \mid \hat{\mathbf{x}}_{1}, \hat{\mathbf{x}}_{2}, \Psi_{F}\right)=p\left(\mathbf{y}_{r d}-\sqrt{\alpha P^{2} \lambda_{r d} \lambda_{s r}} g \sum_{k=1}^{2} f_{k} \hat{\mathbf{x}}_{k},\left(2 \alpha P \lambda_{r d}|g|^{2}+1\right) \mathbf{I}\right) \\
& L\left(\mathbf{y}_{r d} \mid \hat{\mathbf{x}}_{1}, \hat{\mathbf{x}}_{2}, \Psi_{P}\right)=p\left(\mathbf{y}_{r d}, \alpha P^{2} \lambda_{r d} \lambda_{s r}|g|^{2} \sum_{k=1}^{2} \hat{\mathbf{x}}_{k} \hat{\mathbf{x}}_{k}^{H}+\left(2 \alpha P \lambda_{r d}|g|^{2}+1\right) \mathbf{I}\right)
\end{aligned}
$$

the destination while the two source nodes remain silent. The received signal is given by

$$
\mathbf{y}_{r d}=\sqrt{P \lambda_{r d}} g \mathbf{x}_{\mathbf{r}}+\mathbf{n}_{r d}
$$

where $\mathbf{n}_{r d}$ is the additive noises. Upon observing $\mathbf{y}_{r d}$ and $\mathbf{y}_{k d}$ for $k=1,2$, the destination performs ML detection to jointly detect the two source symbols as

$$
\begin{aligned}
\left(\mathbf{x}_{d, 1}, \mathbf{x}_{d, 2}\right)=\arg \max _{\hat{\mathbf{x}}_{1}, \hat{\mathbf{x}}_{2} \in \Omega} L & \left(\mathbf{y}_{r d} \mid \hat{\mathbf{x}}_{1}, \hat{\mathbf{x}}_{2}, \Psi\right) \\
& \times \prod_{k=1}^{2} L\left(\mathbf{y}_{k d} \mid \hat{\mathbf{x}}_{1}, \hat{\mathbf{x}}_{2}, \Psi\right),
\end{aligned}
$$

where $L(\cdot)$ is the corresponding likelihood function, and $\Psi$ is the set of instantaneous CSI available at the destination. For coherent detection, we have $\Psi_{F}=\left\{f_{1}, f_{2}, h_{1}, h_{2}, g\right\}$, i.e., the global CSI is supposed to be known at the destination. Alternatively, the destination can choose to perform noncoherent detection with $\Psi_{S}$ being the empty set, such that channel estimation is no longer required and all the real-time channel variations are blind to the whole network. To facilitate explanations, we also define the partial coherent detection, where the destination only knows the relay-destination channel, i.e., $\Psi_{P}=\{g\}$. As the channel gains (i.e., $\lambda$ ) are only second-order statistics that remain unchanged over a long time, we assume these coefficients are known to all the nodes in the network. According to these definitions, we have

$$
\begin{aligned}
& L\left(\mathbf{y}_{k d} \mid \hat{\mathbf{x}}_{k}, \Psi\right) \\
& \quad=\left\{\begin{array}{l}
p\left(\mathbf{y}_{k d}-\sqrt{P \lambda_{s d}} h_{k} \hat{\mathbf{x}}_{k}, \mathbf{I}\right), \Psi=\Psi_{F} \\
p\left(\mathbf{y}_{k d}, P \lambda_{s d} \hat{\mathbf{x}}_{k} \hat{\mathbf{x}}_{k}^{H}+\mathbf{I}\right), \Psi \in\left\{\Psi_{P}, \Psi_{S}\right\}
\end{array}\right.
\end{aligned}
$$

for $k=1,2$, where $\mathbf{I}$ is the identity matrix and $p(\mathbf{y}, \mathbf{\Sigma})=$ $\frac{1}{\pi^{M}|\boldsymbol{\Sigma}|} \exp \left(-\mathbf{y}^{H} \boldsymbol{\Sigma}^{-1} \mathbf{y}\right)$ is the PDF of $\mathbf{y} \sim \mathcal{C N}(\mathbf{0}, \boldsymbol{\Sigma})$. The likelihood function of the relay link signal $\mathbf{y}_{r d}$ is related to the network coding schemes and would be detailed later.

\section{TRANSCEIVER DESIGN}

In this section, we study the relay operation and the ML detection at the destination under different network coding schemes.

\section{A. Analog Network Coding}

If ANC is used at the relay node, the two received signals $\mathbf{y}_{k r}$ for $k=1,2$ are combined in the complex field directly by $\mathbf{x}_{r}=\sqrt{\alpha}\left(\mathbf{y}_{1 r}+\mathbf{y}_{2 r}\right)$, where

$$
\alpha=\frac{1}{2\left(P \lambda_{s r}+M\right)}
$$

is the amplification factor to normalize the relay power. From (3), the received signal $\mathbf{y}_{r d}$ can then be rewritten as

$$
\begin{aligned}
\mathbf{y}_{r d} & =\sqrt{\alpha P \lambda_{r d}} g \sum_{k=1}^{2}\left(\sqrt{P \lambda_{s r}} f_{k} \mathbf{x}_{k}+\mathbf{n}_{k r}\right)+\mathbf{n}_{r d} \\
& =\sqrt{\alpha P^{2} \lambda_{r d} \lambda_{s r}} g \sum_{k=1}^{2} f_{k} \mathbf{x}_{k}+\tilde{\mathbf{n}}_{r d}
\end{aligned}
$$

where $\tilde{\mathbf{n}}_{r d}=\sqrt{\alpha P \lambda_{r d}} g \sum_{k=1}^{2} \mathbf{n}_{k r}+\mathbf{n}_{r d}$ is the equivalent noises. The likelihood functions of the relay link signal $\mathbf{y}_{r d}$ are then given by (8) and (9) for coherent detection and partial coherent detection, respectively. For non-coherent detection, the likelihood function can be obtained by averaging (9) over the distributions of $g$, i.e.,

$$
L\left(\mathbf{y}_{r d} \mid \hat{\mathbf{x}}_{1}, \hat{\mathbf{x}}_{2}, \Psi_{S}\right)=E_{g}\left[L\left(\mathbf{y}_{r d} \mid \hat{\mathbf{x}}_{1}, \hat{\mathbf{x}}_{2}, \Psi_{P}\right)\right] .
$$

Unfortunately, the above expression is an integral form that has no closed-form solution, which would greatly complicate the implementation. To simplify the receiver design, we develop two suboptimum yet efficient receivers in the sequel. To that end, let us first revisit the signal model (7). The aggregate scaling coefficient effective on the signal component from the input of the relay node to the destination is given by

$$
\sqrt{\alpha P \lambda_{r d}} g=\sqrt{\frac{P \lambda_{r d}}{2\left(P \lambda_{s r}+M\right)}} g \stackrel{P \gg 1}{\approx} \sqrt{\frac{\lambda_{r d}}{2 \lambda_{s r}}} g .
$$

When the source-relay channel is much better than the relaydestination channel (i.e., $\lambda_{r d} \ll \lambda_{s r}$ ), the above scaling coefficient remains small with large probability, whereas the noise power of $\mathbf{n}_{r d}$ is a constant. As a result, we can approximately neglect the fading of $g$ and obtain

$$
L\left(\mathbf{y}_{r d} \mid \hat{\mathbf{x}}_{1}, \hat{\mathbf{x}}_{2}, \Psi_{S}\right) \approx L\left(\mathbf{y}_{r d} \mid \hat{\mathbf{x}}_{1}, \hat{\mathbf{x}}_{2}, \Psi_{P}, g=1\right),
$$

which is called fading elimination approximation (FEA). The error performance is expected to remain similar because the channel fading only brings very limited effects when the scaling coefficient is small on average. On the other hand, if the source-relay channel is much worse than the relay-destination channel (i.e., $\lambda_{r d} \gg \lambda_{s r}$ ), then the noises $\mathbf{n}_{r d}$ would have much lower power than the scaled noises $\sqrt{\alpha P \lambda_{r d}} g \mathbf{n}_{k r}$. Consequently, we can intentionally neglect $\mathbf{n}_{r d}$ and obtain

$$
\mathbf{y}_{r d} \approx \tilde{\mathbf{y}}_{r d}=\sqrt{\alpha P \lambda_{r d}} g \sum_{k=1}^{2}\left(\sqrt{P \lambda_{s r}} f_{k} \mathbf{x}_{k}+\mathbf{n}_{k r}\right) .
$$

To obtain the likelihood function of $\tilde{\mathbf{y}}_{r d}$, we first prove the following lemma. 


$$
P_{r, k}=\left\{\begin{array}{l}
1-\frac{1}{\sqrt{2 \pi}} \int_{-\infty}^{\infty}(1-Q(x))^{M-1} e^{-\frac{1}{2}\left(x-\sqrt{2 P \lambda_{s r}\left|f_{k}\right|^{2}}\right)^{2}} d x, \Psi=\Psi_{F} \\
\sum_{k=1}^{M-1}\left(\begin{array}{c}
M-1 \\
k
\end{array}\right) \frac{(-1)^{k+1}}{1+k\left(1+P \lambda_{s r}\right)}, \Psi=\Psi_{S}
\end{array}\right.
$$

Lemma 1: Suppose $\mathbf{v} \sim \mathcal{C N}\left(\mathbf{0}, \operatorname{diag}\left\{\sigma_{v_{i}}^{2}\right\}_{i=1}^{M}\right)$ and $u \sim$ $\mathcal{C N}\left(0, \sigma_{u}^{2}\right)$ are independent random variables, then the PDF of $\mathbf{z}=u \mathbf{v}$ is

$$
f(\mathbf{z})=\left(\prod_{i=1}^{M} \frac{2}{\pi \sigma_{u}^{2} \sigma_{v_{i}}^{2}}\right) q\left(\frac{4}{\sigma_{u}^{2}} \sum_{i=1}^{M} \frac{\left|z_{i}\right|^{2}}{\sigma_{v_{i}}^{2}}\right),
$$

where $q(x)=x^{-\frac{M-1}{2}} K_{M-1}(\sqrt{x})$ and $K_{M}(x)$ is the $M$ thorder modified Bessel function of the second kind [23, 9.6.1].

Proof: Denote $z_{i}=r_{i} e^{j \theta_{i}}$ for $i=1,2, \cdots, M$, then it is easy to show that the phases $\left\{\theta_{i}\right\}$ are independent of the amplitudes $\left\{r_{i}\right\}$, and $\left\{\theta_{i}\right\}$ are i.i.d. and uniformly distributed on $[0,2 \pi)$. Therefore,

$$
f(\mathbf{z})=\frac{1}{|J|} f(\mathbf{r}, \boldsymbol{\theta})=\prod_{i=1}^{M}\left(2 \pi r_{i}\right)^{-1} f(\mathbf{r}),
$$

where $|J|=\prod_{i=1}^{M} r_{i}$ is the Jacobian determinant. The CDF of $\mathbf{r}$ is given by

$$
F(\mathbf{r})=\int_{0}^{\infty} \frac{1}{\sigma_{u}^{2}} \exp \left(-\frac{x}{\sigma_{u}^{2}}\right) \prod_{i=1}^{M}\left(1-\exp \left(-\frac{r_{i}^{2}}{\sigma_{v_{i}}^{2} x}\right)\right) d x .
$$

After taking derivatives, we can obtain

$$
\begin{aligned}
f(\mathbf{r}) & =\frac{1}{\sigma_{u}^{2}} \prod_{i=1}^{M} \frac{2 r_{i}}{\sigma_{v_{i}}^{2}} \int_{0}^{\infty} x^{-M} \exp \left(-\frac{x}{\sigma_{u}^{2}}-\frac{1}{x} \sum_{i=1}^{M} \frac{r_{i}^{2}}{\sigma_{v_{i}}^{2}}\right) d x \\
& =\left(\prod_{i=1}^{M} \frac{4 r_{i}}{\sigma_{u}^{2} \sigma_{v_{i}}^{2}}\right) q\left(\frac{4}{\sigma_{u}^{2}} \sum_{i=1}^{M} \frac{r_{i}^{2}}{\sigma_{v_{i}}^{2}}\right),
\end{aligned}
$$

where we use $[25,3.478 .4]$ in the last equality. Plugging (17) back into (15) completes the proof.

According to Lemma 1, the likelihood function of $\tilde{\mathbf{y}}_{r d}$ can be obtained after redefining the parameters in (14). To be specific, for $\hat{\mathbf{x}}_{1}=\hat{\mathbf{x}}_{2}=\mathbf{e}_{l}$ we have $\sigma_{u}^{2}=\alpha P \lambda_{r d}$, $\sigma_{v_{l}}^{2}=2\left(P \lambda_{s r}+1\right)$ and $\sigma_{v_{i}}^{2}=2$ for $i \neq l$, whereas for $\hat{\mathbf{x}}_{1}=\mathbf{e}_{k}, \hat{\mathbf{x}}_{2}=\mathbf{e}_{l}$ with $k \neq l$ we have $\sigma_{u}^{2}=\alpha P \lambda_{r d}$, $\sigma_{v_{k}}^{2}=\sigma_{v_{l}}^{2}=P \lambda_{s r}+2$ and $\sigma_{v_{i}}^{2}=2$ for $i \neq l, k$. In later sections, this scheme is referred to as noise elimination approximation (NEA).

\section{B. Digital Network Coding}

For DNC, the two source symbols are first individually detected at the relay node as

$$
\mathbf{x}_{r, k}=\left\{\begin{array}{l}
\arg \max _{\hat{\mathbf{x}}_{k} \in \Omega} p\left(\mathbf{y}_{k r}-\sqrt{P \lambda_{s r}} f_{k} \hat{\mathbf{x}}_{k}, \mathbf{I}\right), \Psi=\Psi_{F}(18 \mathrm{a}) \\
\arg \max _{\hat{\mathbf{x}}_{k} \in \Omega} p\left(\mathbf{y}_{k r}, P \lambda_{s r} \hat{\mathbf{x}}_{k} \hat{\mathbf{x}}_{k}^{H}+\mathbf{I}\right), \Psi=\Psi_{S}
\end{array}\right.
$$

where the relay node is assumed to know the same type of CSI as the destination. Next, the detected source symbols are combined in the finite field, and the relay symbol is given by $\mathbf{x}_{r}=\mathbf{x}_{r, 1} \oplus \mathbf{x}_{r, 2}$. We remark that the conventional DNC is performed bitwise through XOR operations [3]. Here to simplify the notations we omit the mapping between the bits and symbols and express the network-coded output in the symbol space directly. It is also noteworthy that $\mathbf{x}_{r}$ belongs to the M-ary FSK symbol set $\Omega$ as well, but it maybe different from the true network-coded symbol $\mathbf{x}_{\oplus}=\mathbf{x}_{1} \oplus \mathbf{x}_{2}$ due to random detection errors.

The likelihood function of the relay link signal $\mathbf{y}_{r d}$ is

$$
\begin{aligned}
& L\left(\mathbf{y}_{r d} \mid \hat{\mathbf{x}}_{1}, \hat{\mathbf{x}}_{2}, \Psi\right)= \\
& \quad \sum_{k=1}^{M} \operatorname{Pr}\left(\hat{\mathbf{x}}_{r}=\mathbf{e}_{k} \mid \hat{\mathbf{x}}_{1}, \hat{\mathbf{x}}_{2}, \Psi\right) L\left(\mathbf{y}_{r d} \mid \hat{\mathbf{x}}_{r}=\mathbf{e}_{k}, \Psi\right),
\end{aligned}
$$

where the second term within the summation has the same form as (5) after properly modifying the subscripts, and the first term is given by

$$
\begin{aligned}
\operatorname{Pr} & \left(\hat{\mathbf{x}}_{r}=\mathbf{e}_{k} \mid \hat{\mathbf{x}}_{1}, \hat{\mathbf{x}}_{2}, \Psi\right) \\
\quad & =\sum_{\hat{\mathbf{x}}_{r, 1} \oplus \hat{\mathbf{x}}_{r, 2}=\mathbf{e}_{k}} \operatorname{Pr}\left(\hat{\mathbf{x}}_{r, k} \mid \hat{\mathbf{x}}_{1}, \Psi\right) \operatorname{Pr}\left(\hat{\mathbf{x}}_{r, 2} \mid \hat{\mathbf{x}}_{2}, \Psi\right) .
\end{aligned}
$$

Note that each term within the summation stands for the transition probability from the trial source symbol $\hat{\mathbf{x}}_{k}$ to the trial relay detected symbol $\hat{\mathbf{x}}_{r, k}$. For FSK modulations, the error rates at the relay node can be obtained from [24, 5.2.19] and $[24,5.4 .42]$ after properly accommodating the channel fading and are respectively given by (21a) and (21b), where $Q(x)$ is Q-function. Due to symmetry, the detection errors of FSK modulations are uniformly distributed in the error symbol set, so each transition probability is given by

$$
\operatorname{Pr}\left(\hat{\mathbf{x}}_{r, k} \mid \hat{\mathbf{x}}_{k}, \Psi\right)=\left\{\begin{array}{l}
\frac{1}{M-1} P_{r, k}, \hat{\mathbf{x}}_{r, k} \neq \hat{\mathbf{x}}_{k} \\
1-P_{r, k}, \hat{\mathbf{x}}_{r, k}=\hat{\mathbf{x}}_{k}
\end{array}\right.
$$

for $k=1,2$. Note that for coherent detection, the destination knows the instantaneous relay detection error rates, whereas for non-coherent detection only the average relay detection error rates are supposed to be known at the destination.

\section{Error Performance Analysis}

In this section, we study the error rates of the aforementioned systems. Unfortunately, the exact error rates are analytically intractable due to the complex ML decision regions. Instead, we study the PEPs, which are defined as the probability of mistaking the true symbols $\left(\mathbf{x}_{1}, \mathbf{x}_{2}\right)$ by another trial symbols $\left(\hat{\mathbf{x}}_{1}, \hat{\mathbf{x}}_{2}\right)$. It is well known that the real error rates can be approximately characterized by the dominant PEPs [1]. Without loss of generality, we focus only on the binary FSK modulations throughout this section. Due to symmetry, there are four types of PEPs as listed in Table I. 


$$
\left.\mathrm{PEP}\right|_{\Psi_{F}}=Q\left(\sqrt{\frac{1}{2}\left(P \lambda_{s d} \sum_{k=1}^{2}\left|h_{k}\right|^{2}\left\|\mathbf{x}_{k}-\hat{\mathbf{x}}_{k}\right\|^{2}+\frac{\alpha P^{2} \lambda_{r d} \lambda_{s r}|g|^{2}}{1+2 \alpha P \lambda_{r d}|g|^{2}}\left\|\sum_{k=1}^{2} f_{k}\left(\mathbf{x}_{k}-\hat{\mathbf{x}}_{k}\right)\right\|^{2}\right)}\right)
$$

$$
\mathrm{PEP} \geq \frac{(2 n+1) ! !}{(2 n+2) ! !} \frac{2^{n-1}}{\left(2+P \lambda_{s d}\right)^{n}}\left(\frac{4}{4+n P \lambda_{s r}}+\frac{n \lambda_{s r}}{\alpha \lambda_{r d}\left(4+n P \lambda_{s r}\right)^{2}} \log \left(1+4 \alpha P \lambda_{r d}+\alpha n P^{2} \lambda_{r d} \lambda_{s r}\right)\right)
$$

$$
\mathrm{PEP} \leq \frac{2^{n-1}}{\left(2+P \lambda_{s d}\right)^{n}}\left(\frac{4}{4+n P \lambda_{s r}}+\frac{2 n P \lambda_{s r}}{\alpha P \lambda_{r d}\left(4+n P \lambda_{s r}\right)^{2}} \log \left(1+2 \alpha P \lambda_{r d}+\frac{1}{2} \alpha n P^{2} \lambda_{r d} \lambda_{s r}\right)\right)
$$

TABLE I

FOUR TYPES OF PEP

\begin{tabular}{c|c|c}
\hline Notations & True Symbols & Trial Symbols \\
\hline$P_{1}$ & $\left(\mathbf{e}_{1}, \mathbf{e}_{1}\right)$ & $\left(\mathbf{e}_{2}, \mathbf{e}_{2}\right)$ \\
$P_{2}$ & $\left(\mathbf{e}_{1}, \mathbf{e}_{2}\right)$ & $\left(\mathbf{e}_{2}, \mathbf{e}_{1}\right)$ \\
$P_{3}$ & $\left(\mathbf{e}_{1}, \mathbf{e}_{1}\right)$ & $\left(\mathbf{e}_{1}, \mathbf{e}_{2}\right)$ \\
$P_{4}$ & $\left(\mathbf{e}_{1}, \mathbf{e}_{2}\right)$ & $\left(\mathbf{e}_{1}, \mathbf{e}_{1}\right)$ \\
\hline
\end{tabular}

\section{A. Coherent Analog Network Coding}

After some manipulations, we can obtain the conditional PEPs given by (23). To obtain the average PEPs, we need to average the above expression over the channel distributions, which is analytically intractable. Instead, we seek to develop some bounds that have the same scaling laws as the true PEPs at high SNRs. To that end, we first give the following lemma.

Lemma 2: Let $W=\frac{a b X Y}{a X+c}$, where $a, b$ and $c$ are constants, $X \sim \exp (1)$ and $Y \sim \exp (1)$ are independent. Then the CDF of $W$ and the expectation of $\exp (-W)$ are respectively given by

$$
\begin{aligned}
& F_{W}(w)=1-\exp \left(-\frac{w}{b}\right) \sqrt{\frac{4 w c}{a b}} K_{1}\left(\sqrt{\frac{4 w c}{a b}}\right) \\
& \ll 1 \frac{c}{a b} w \log \frac{1}{w}, \\
& E[\exp (-W)]=\frac{1}{b+1} \\
&+\frac{b c}{a(b+1)^{2}} \exp \left(\frac{c}{a(b+1)}\right) E_{1}\left(\frac{c}{a(b+1)}\right),
\end{aligned}
$$

where $E_{1}(z)$ is the exponential integral function $[23,5.1 .1]$ and $K_{1}(z)$ is the first-order modified Bessel function of the second kind [23, 9.6.1].

Proof: See [8, 9].

To obtain the PEP lower bound, we use the integral representation of Q-function given by [1]

$$
Q(x)=\frac{1}{\pi} \int_{0}^{\frac{\pi}{2}} \exp \left(-\frac{x^{2}}{2 \sin ^{2} \theta}\right) d \theta .
$$

After replacing the Q-function in (23) with the above expression and averaging over the channel distributions by use of (25), we can obtain (27), where $(\cdot) ! !$ is double factorial, $n=$ $\mathbb{1}\left\{\mathbf{x}_{1} \neq \hat{\mathbf{x}}_{1}\right\}+\mathbb{1}\left\{\mathbf{x}_{2} \neq \hat{\mathbf{x}}_{2}\right\}$ with $\mathbb{1}\{\cdot\}$ being indicator function, and we use $\frac{1}{2} \log \left(1+\frac{2}{x}\right)<e^{x} E_{1}(x)$ [23, 5.1.20] in the inequality. To obtain the PEP upper bound, we use the Chernoff bound of Q-function given by $Q(x) \leq \frac{1}{2} \exp \left(-\frac{1}{2} x^{2}\right)$
[1]. In a similar way, we can obtain (28), where we use $e^{x} E_{1}(x)<\log \left(1+\frac{1}{x}\right)[23,5.1 .20]$ in the inequality. From (23), it is observed that Type-1 PEP and Type-2 PEP have the same conditional distributions, and Type-3 PEP and Type- 4 PEP also have the same conditional distributions. That is why the PEP bounds (27) and (28) depend only on $n$ but not the true and trial symbol pairs. At high SNRs (i.e. $P \gg 1$ ), we can show that both the upper bound and lower bound scale as $\mathrm{O}\left(\frac{\log P}{P^{n+1}}\right)$. Clearly, the dominant error events occur when only one of the two source symbols is detected incorrectly at the destination (i.e., $n=1$ ), and the error rates of coherent ANC scale as $\mathrm{O}\left(\frac{\log P}{P^{2}}\right)$ at high SNRs.

\section{B. Non-Coherent Analog Network Coding}

Since the non-coherent ML receiver (10) has an intractable integral form, we study the partial coherent receiver (9). As will be shown in simulations, the performances of these two receivers are very close to each other.

For Type-1 PEP, we have

$$
\begin{gathered}
P_{1}=\operatorname{Pr}\left\{\frac{\lambda_{s d} P}{1+\lambda_{s d} P}\left(U_{2}^{1}+V_{2}^{1}\right)+\frac{\lambda_{s r} P Q}{1+\lambda_{s r} P Q} W_{2}^{1}\right. \\
\left.\geq \lambda_{s d} P\left(U_{1}^{1}+V_{1}^{1}\right)+\lambda_{s r} P Q W_{1}^{1}\right\} \\
\leq \operatorname{Pr}\left\{U_{2}^{1}+V_{2}^{1}+W_{2}^{1} \geq P Y\right\},
\end{gathered}
$$

where $W_{1}^{1}=\frac{\left|y_{r d, 1}\right|^{2}}{1+2 \alpha P \lambda_{r d}|g|^{2}+2 \alpha P^{2} \lambda_{r d} \lambda_{s r}|g|^{2}}, U_{1}^{1}=\frac{\left|y_{1 d, 1}\right|^{2}}{1+\lambda_{s d} P}$, $V_{1}^{1}=\frac{\left|y_{2 d, 1}\right|^{2}}{1+\lambda_{s d} P}, W_{2}^{1}=\frac{\left|y_{r d, 2}\right|^{2} \lambda_{r d} \lambda_{s r}|g|}{1+2 \alpha P \lambda_{r d}|g|^{2}}, U_{2}^{1}=\left|y_{1 d, 2}\right|^{2}, V_{2}^{1}=$ $\left|y_{2 d, 2}\right|^{2}$ are i.i.d. exponential random variables with unit mean, and

$$
Q=\frac{2 \alpha P \lambda_{r d}|g|^{2}}{1+2 \alpha P \lambda_{r d}|g|^{2}}
$$

Since $U_{2}^{1}+V_{2}^{1}+W_{2}^{1} \sim \frac{1}{2} \chi_{6}^{2}$, the conditional PEP given $Y \triangleq$ $\lambda_{s d}\left(U_{1}^{1}+V_{1}^{1}\right)+\lambda_{s r} Q W_{1}^{1}=y$ is

$$
\left.P_{1}\right|_{Y=y} \leq\left(1+P y+\frac{1}{2} P^{2} y^{2}\right) \exp (-P y) .
$$

At high SNRs, the conditional PEP decreases really fast with $y$. Therefore, the average PEP is roughly determined by the behavior of the PDF of $Y \ll 1$. Denoting $T \triangleq Q W_{1}^{1}$, we can obtain

$$
F_{Y}(y) \stackrel{y \ll 1}{\approx} \int_{0}^{\frac{y}{\lambda_{s r}}} f_{T}(t) \frac{\left(y-\lambda_{s r} t\right)^{2}}{\lambda_{s d}^{2}} d t
$$




$$
P_{4}^{U_{1}}=\left(1+\eta_{2}\right) e^{-\eta_{2}}-\left(\frac{\lambda_{s d} P}{2+\lambda_{s d} P}\right)^{2}\left(1+\frac{2+\lambda_{s d} P}{\lambda_{s d} P} \eta_{2}\right) e^{-\frac{2+\lambda_{s d} P}{\lambda_{s d} P} \eta_{2}}=\mathrm{O}\left(\frac{\log ^{2} P}{P^{2}}\right)
$$

Taking derivative with respect to $y$ leads to

$$
\begin{aligned}
f_{Y}(y) & \stackrel{y \ll 1}{\approx} \frac{2 \lambda_{s r}}{\lambda_{s d}^{2}} \int_{0}^{\frac{y}{\lambda_{s r}}} F_{T}(t) d t \\
& =-\frac{y^{2}}{2 \alpha P \lambda_{s r} \lambda_{r d} \lambda_{s d}^{2}}\left(\log \frac{y}{\lambda_{s r}}-\frac{1}{2}\right),
\end{aligned}
$$

where we use $F_{T}(t) \stackrel{t \ll 1}{\approx}-\frac{1}{2 \alpha P \lambda_{r d}} t \log t$ from (24) in the second equality. Using the above PDF to average the conditional PEP in (31), we obtain

$$
P_{1} \leq \frac{20 \log \left(\sqrt{e} P \lambda_{s r}\right)-39+20 \gamma}{2 \alpha P^{4} \lambda_{s r} \lambda_{r d} \lambda_{s d}^{2}}=\mathrm{O}\left(\frac{\log P}{P^{3}}\right),
$$

where $\gamma$ is Euler constant [25, 4.352.2].

For Type-2 PEP, the likelihood function of $\mathbf{y}_{r d}$ remains the same under both hypotheses, which greatly simplifies the computations. After some manipulations, we can obtain

$$
\begin{aligned}
P_{2} & =\operatorname{Pr}\left\{\left|y_{1 d, 2}\right|^{2}+\left|y_{2 d, 1}\right|^{2} \geq\left|y_{1 d, 1}\right|^{2}+\left|y_{2 d, 2}\right|^{2}\right\} \\
& =\frac{4+3 \lambda_{s d} P}{\left(2+\lambda_{s d} P\right)^{3}}=\mathrm{O}\left(\frac{1}{P^{2}}\right) .
\end{aligned}
$$

For Type-3 PEP, we have

$$
\begin{aligned}
P_{3}= & \operatorname{Pr}\left\{\frac{\lambda_{s d} P}{1+\lambda_{s d} P} V_{2}^{3}+\frac{\lambda_{s r} P Q}{2+\lambda_{s r} P Q} W_{2}^{3}\right. \\
& \left.\geq \lambda_{s d} P V_{1}^{3}+\frac{\lambda_{s r} P Q}{2+\lambda_{s r} P Q} W_{1}^{3}+\log \frac{\left(2+\lambda_{s r} P Q\right)^{2}}{4\left(1+\lambda_{s r} P Q\right)}\right\} \\
\leq & \operatorname{Pr}\left\{W_{2}^{3}+V_{2}^{3} \geq \lambda_{s d} P V_{1}^{3}+\log Z\right\},
\end{aligned}
$$

where $W_{1}^{3}=\frac{\left|y_{r d, 1}\right|^{2}}{1+2 \alpha P \lambda_{r d}|g|^{2}+2 \alpha P^{2} \lambda_{r d} \lambda_{s r}|g|^{2}}, V_{1}^{3}=\frac{\left|y_{2 d, 1}\right|^{2}}{1+\lambda_{s d} P}$, $W_{2}^{3}=\frac{\left|y_{r d, 2}\right|^{2}}{1+2 \alpha P \lambda_{r d}|g|^{2}}$, and $V_{2}^{3}=\left|y_{2 d, 2}\right|^{2}$ are i.i.d. exponential random variables with unit mean, and $Z=\frac{2+\lambda_{s r} P Q}{4} \leq \frac{1}{2}+$ $\frac{P \lambda_{s r}}{4}$. The conditional PEP given $Z=z$ is given by

$$
\left.P_{3}\right|_{Z=z} \leq \frac{\left(1+2 \lambda_{s d} P\right)+\left(1+\lambda_{s d} P\right) \log \left(\frac{1}{2}+\frac{P \lambda_{s r}}{4}\right)}{\left(1+\lambda_{s d} P\right)^{2} z} .
$$

By defining $\eta_{1}=\alpha P \lambda_{r d}\left(2+\lambda_{s r} P\right)$, we can further obtain

$E\left(\frac{1}{Z}\right)=\frac{1}{\eta_{1}}\left(4 \alpha \lambda_{r d} P+\frac{2 \lambda_{s r} P}{2+\lambda_{s r} P} \exp \left(\frac{1}{\eta_{1}}\right) E_{1}\left(\frac{1}{\eta_{1}}\right)\right)$.

After combining (37) and (38) and using the inequality $e^{x} E_{1}(x) \leq \log \left(1+\frac{1}{x}\right)[23,5.1 .20]$, we obtain $P_{3} \leq$ $\mathrm{O}\left(\frac{\log ^{2} P}{P^{2}}\right)$

Finally for Type-4 PEP, we have

$$
\begin{aligned}
& P_{4}=\operatorname{Pr}\left\{\frac{\lambda_{s d} P}{1+\lambda_{s d} P} V_{1}^{4}+\frac{\lambda_{s r} P Q}{2\left(1+\lambda_{s r} P Q\right)} W_{1}^{4}\right. \\
& \left.+\log \frac{\left(2+\lambda_{s r} P Q\right)^{2}}{4\left(1+\lambda_{s r} P Q\right)} \geq \lambda_{s d} P V_{2}^{4}+\frac{1}{2} \lambda_{s r} P Q W_{2}^{4}\right\},
\end{aligned}
$$

where $W_{1}^{4}=\frac{\left|y_{r d, 1}\right|^{2}}{1+2 \alpha P \lambda_{r d}|g|^{2}+\alpha P^{2} \lambda_{r d} \lambda_{s r}|g|^{2}}, \quad V_{1}^{4}=\left|y_{2 d, 1}\right|^{2}$, $W_{2}^{4}=\frac{\left|y_{r d, 2}\right|^{2}}{1+2 \alpha P \lambda_{r d}|g|^{2}+\alpha P^{2} \lambda_{r d} \lambda_{s r}|g|^{2}}, V_{2}^{4}=\frac{\left|y_{2 d, 2}\right|^{2}}{1+\lambda_{s d} P}$ are i.i.d. exponential random variables with unit mean. As the logarithmic term is upper bounded by $\log \frac{\left(2+\lambda_{s r} P\right)^{2}}{4\left(1+\lambda_{s r} P\right)} \triangleq \eta_{2}$, we have

$$
\begin{gathered}
P_{4} \leq \underbrace{\operatorname{Pr}\left\{W_{1}^{I V}+V_{1}^{I V} \geq \max \left\{\frac{1}{2} \lambda_{s d} P V_{2}^{I V}, \eta_{2}\right\}\right\}}_{\triangleq P_{4}^{U_{1}}} \\
+\underbrace{\operatorname{Pr}\left\{2 \eta_{2} \geq \lambda_{s d} P V_{2}^{I V}+\frac{1}{2} \lambda_{s r} P Q W_{2}^{I V}\right\}}_{\triangleq P_{4}^{U_{2}}} .
\end{gathered}
$$

After some lengthy algebra, we can obtain (41) and

$$
\begin{aligned}
P_{4}^{U_{2}} \stackrel{P}{ } & \gtrsim \frac{\eta_{2}^{2}}{\alpha P^{3} \lambda_{s r} \lambda_{s d} \lambda_{r d}}\left(1-2 \log \left(\frac{4 \eta_{2}}{\lambda_{s r} P}\right)\right) \\
& =\mathrm{O}\left(\frac{\log ^{3} P}{P^{2}}\right) .
\end{aligned}
$$

Consequently, we have $P_{4} \leq \mathrm{O}\left(\frac{\log ^{3} P}{P^{2}}\right)$, which appears to dominate all the PEPs. To make the argument rigorous, yet we still need to show that this is the exact scaling law of Type-4 PEP. To that end, we develop a lower bound on $P_{4}$ by neglecting the first two terms on the left-hand side of the inequality in (39), which leads to

$$
\begin{aligned}
P_{4} & \geq \operatorname{Pr}\left\{\log \frac{2+\lambda_{s r} P Q}{4} \geq \lambda_{s d} P V_{2}^{4}+\frac{1}{2} \lambda_{s r} P Q W_{2}^{4}\right\} \\
& \triangleq P_{4}^{L} .
\end{aligned}
$$

The conditional probability of $P_{4}^{L}$ given $Q=q$ is given by

$$
\begin{aligned}
\left.P_{4}^{L}\right|_{Q=q} & =1-\frac{2 \lambda_{s d}}{2 \lambda_{s d}-q \lambda_{s r}} \exp \left(-\frac{1}{\lambda_{s d} P} \log \frac{2+q \lambda_{s r} P}{4}\right) \\
& +\frac{q \lambda_{s r}}{2 \lambda_{s d}-q \lambda_{s r}} \exp \left(-\frac{2}{q \lambda_{s r} P} \log \frac{2+q \lambda_{s r} P}{4}\right) .
\end{aligned}
$$

When $q \geq P^{-\beta}$ for any constant $\beta \in(0,1)$, we have $q P \geq$ $P^{1-\beta} \gg 1$ for $P \gg 1$, thus

$$
\left.P_{4}^{L}\right|_{Q=q} \stackrel{P \gg 1}{\approx} \frac{1}{q \lambda_{s r} \lambda_{s d} P^{2}}\left(\log \frac{2+q \lambda_{s r} P}{4}\right)^{2} .
$$

The final step is to average the above expression over the distribution of $Q$. The PDF of $Q$ is given by

$$
f_{Q}(q)=\frac{1}{2 \alpha P \lambda_{r d}(1-q)^{2}} \exp \left(-\frac{q}{2 \alpha P \lambda_{r d}(1-q)}\right)
$$

for $0 \leq q \leq 1$. It is easy to see that $f_{Q}(q)$ is a continuous function with $f_{Q}(0)=\frac{1}{2 \alpha P \lambda_{r d}}$ and $f_{Q}(1)=0$, therefore it is lower bounded by some constant $C$ on the region $q \in\left[0, \eta_{3}\right]$ with $\eta_{3}<1$ being some fixed number. Using the above facts, 


$$
P_{r}=\prod_{k=1}^{2} Q\left(\sqrt{P \lambda_{s r}\left|f_{k}\right|^{2}}\right)\left(1-Q\left(\sqrt{P \lambda_{s r}\left|f_{\{1,2\} \backslash\{k\}}\right|^{2}}\right)\right) \triangleq P_{r, F}
$$

$$
h_{0}(a, b, c, d)=\frac{a}{a+b}\left\{1+\frac{b c}{(c+d)(a+d)}-\frac{b d}{(c+d)(b+c)}\right\}
$$

we can obtain

$$
\begin{aligned}
P_{4}^{L} & \geq \int_{P^{-\beta}}^{\eta_{3}} \frac{1}{q \lambda_{s r} \lambda_{s d} P^{2}}\left(\log \frac{2+q \lambda_{s r} P}{4}\right)^{2} f_{Q}(q) d q \\
& \geq \frac{C \log \left(\eta_{3} P^{\beta}\right)}{\lambda_{s r} \lambda_{s d} P^{2}}\left(\log \frac{2+\lambda_{s r} P^{1-\beta}}{4}\right)^{2} \\
& =\mathrm{O}\left(\frac{\log ^{3} P}{P^{2}}\right)
\end{aligned}
$$

As the upper bound and lower bound on $P_{4}$ have exactly the same scaling laws, we conclude that the error rates of partial coherent ANC scale as $\mathrm{O}\left(\frac{\log ^{3} P}{P^{2}}\right)$ at high SNRs.

\section{Digital Network Coding}

For DNC, we assume $\mathbf{x}_{1} \oplus \mathbf{x}_{2}=\mathbf{e}_{1}$ for $\mathbf{x}_{1}=\mathbf{x}_{2}$ and $\mathbf{x}_{1} \oplus \mathbf{x}_{2}=\mathbf{e}_{2}$ for $\mathbf{x}_{1} \neq \mathbf{x}_{2}$. The relay symbol $\mathbf{x}_{r}=\mathbf{x}_{r, 1} \oplus \mathbf{x}_{r, 2}$ is a single binary FSK symbol that carries information for both sources. Thus the likelihood function of the relay link signal $\mathbf{y}_{r d}$ depends only on the true network-coded symbol $\mathbf{x}_{\oplus}$ rather than the source symbol pair $\left(\mathbf{x}_{1}, \mathbf{x}_{2}\right)$. From Table I, we can observe that regardless of the type of receiver, Type-1 PEP and Type-2 PEP are always the same, and Type-3 PEP and Type4 PEP are the same as well. So in the sequel, we only study Type-1 PEP and Type-3 PEP, in which cases the true source symbols are $\left(\mathbf{e}_{1}, \mathbf{e}_{1}\right)$ and the true network-coded symbol is $\mathbf{x}_{\oplus}=\mathbf{e}_{1} \oplus \mathbf{e}_{1}=\mathbf{e}_{1}$.

To proceed, we first define

$$
t_{k d}=\left\{\begin{array}{l}
2 \sqrt{P \lambda_{s d}} \operatorname{Re}\left\{h_{k}^{*}\left(y_{k d, 1}-y_{k d, 2}\right)\right\}, \Psi=\Psi_{F} \\
\frac{P \lambda_{s d}}{1+P \lambda_{s d}}\left(\left|y_{k d, 1}\right|^{2}-\left|y_{k d, 2}\right|^{2}\right), \Psi=\Psi_{S}
\end{array}\right.
$$

for $k=1,2$ and

$$
t_{r d}=\left\{\begin{array}{l}
2 \sqrt{P \lambda_{r d}} \operatorname{Re}\left\{g^{*}\left(y_{r d, 1}-y_{r d, 2}\right)\right\}, \Psi=\Psi_{F} \\
\frac{P \lambda_{r d}}{1+P \lambda_{r d}}\left(\left|y_{r d, 1}\right|^{2}-\left|y_{r d, 2}\right|^{2}\right), \Psi=\Psi_{S}
\end{array}\right.
$$

It can be shown that the above metrics can be rewritten as the quadratic forms of some independent complex Gaussian random variables. According to [26], the PDF of $t_{k d}$ is $f_{t_{k d}}(t)=f\left(a_{s d}, b_{s d}, t\right)$ for $k=1,2$, the conditional PDF of $t_{r d}$ given $\mathbf{x}_{r}=\mathbf{x}_{\oplus}$ is $\left.f_{t_{r d}}(t)\right|_{\mathbf{x}_{r}=\mathbf{x}_{\oplus}}=f\left(a_{r d}, b_{r d}, t\right)$, and the conditional PDF of $t_{r d}$ given $\mathbf{x}_{r} \neq \mathbf{x}_{\oplus}$ is $\left.f_{t_{r d}}(t)\right|_{\mathbf{x}_{r} \neq \mathbf{x}_{\oplus}}=$ $f\left(b_{r d}, a_{r d}, t\right)$, where

$$
f(a, b, t)=\frac{a b}{a+b}(\exp (b t) \mathbb{1}\{t<0\}+\exp (-a t) \mathbb{1}\{t \geq 0\}),
$$

and for $k \in\{s, r\}, b_{k d}=a_{k d}+1$ and

$$
a_{k d}=\left\{\begin{array}{l}
\frac{1}{2}\left(\sqrt{1+\frac{2}{P \lambda_{k d}}}-1\right), \Psi=\Psi_{F} \\
\frac{1}{P \lambda_{k d}}, \Psi=\Psi_{S}
\end{array}\right.
$$
by

After some algebra, we can show that Type-1 PEP is given

$$
P_{1}=\operatorname{Pr}\left(\sum_{k=1}^{2} t_{k d} \leq 0\right)=\frac{a_{s d}^{2}\left(a_{s d}+3 b_{s d}\right)}{\left(a_{s d}+b_{s d}\right)^{3}}
$$

At high SNRs, Type-1 PEP can be approximated by

$$
P_{1} \stackrel{P \gg 1}{\approx}\left\{\begin{array}{l}
\frac{3}{4 P^{2} \lambda_{s d}^{2}}, \text { coherent } \\
\frac{3}{P^{2} \lambda_{s d}^{2}}, \text { non-coherent }
\end{array}\right.
$$

and the scaling law is $P_{1}=\mathrm{O}\left(\frac{1}{P^{2}}\right)$ regardless of the type of the receiver. As for Type-3 PEP, we have

$$
P_{3}=\operatorname{Pr}\left(t_{2 d}+\log \frac{\left(1-P_{r}\right) \mathrm{e}^{t_{r d}}+P_{r}}{P_{r} \mathrm{e}^{t_{r d}}+\left(1-P_{r}\right)} \leq 0\right),
$$

where $P_{r}$ is the relay detection error rate of the networkcoded symbol $\mathbf{x}_{\oplus}$. For coherent detection and non-coherent detection, $P_{r}$ are respectively given by (55) and

$$
P_{r}=\frac{2\left(1+P \lambda_{s r}\right)}{\left(2+P \lambda_{s r}\right)^{2}} \triangleq P_{r, S}
$$

As the exact analysis is intractable, we use the piecewise linear approximation [15] given by

$$
\log \frac{\left(1-P_{r}\right) \mathrm{e}^{t_{r d}}+P_{r}}{P_{r} \mathrm{e}^{t_{r d}}+\left(1-P_{r}\right)} \approx\left\{\begin{array}{l}
T_{r}, t_{r d} \geq T_{r} \\
t_{r d},-T_{r, S} \leq t_{r d} \leq T_{r} \\
-T_{r}, t_{r d} \leq-T_{r}
\end{array}\right.
$$

where $T_{r}=\log \frac{1-P_{r}}{P_{r}}$. After applying the above approximation in (54) and averaging over the channel distributions of $\left\{h_{1}, h_{2}, g\right\}$, we can obtain the conditional Type-3 PEP given $\left\{f_{1}, f_{2}\right\}$ as

$$
\begin{aligned}
\left.P_{3}\right|_{\left\{f_{1}, f_{2}\right\}}= & \left(1-P_{r}\right) h_{0}\left(a_{r d}, b_{r d}, a_{s d}, b_{s d}\right) \\
& +P_{r} h_{0}\left(b_{r d}, a_{r d}, a_{s d}, b_{s d}\right) \\
& -\frac{a_{s d}}{b_{r d}+a_{s d}}\left(1-P_{r}\right) h_{1}\left(b_{r d}, a_{r d}, a_{s d}, b_{s d}, T_{r}\right) \\
& -\frac{a_{s d}}{a_{r d}+a_{s d}} P_{r} h_{1}\left(a_{r d}, b_{r d}, a_{s d}, b_{s d}, T_{r}\right) \\
& +\frac{b_{s d}}{a_{r d}+b_{s d}}\left(1-P_{r}\right) h_{1}\left(a_{r d}, b_{r d}, b_{s d}, a_{s d}, T_{r}\right) \\
& +\frac{b_{s d}}{b_{r d}+b_{s d}} P_{r} h_{1}\left(b_{r d}, a_{r d}, b_{s d}, a_{s d}, T_{r}\right),
\end{aligned}
$$

where $h_{0}(a, b, c, d)$ is given by (59) and

$$
h_{1}(a, b, c, d, t)=\frac{b}{a+b} \frac{d}{c+d} \exp (-(a+c) t) .
$$

For non-coherent detection, (58) is exactly the average Type-3 PEP, because $P_{r, S}$ in (56) is independent of the source-relay 


$$
\begin{aligned}
& P_{3} \approx h_{0}\left(a_{r d}, b_{r d}, a_{s d}, b_{s d}\right)+h_{5}\left(0,1,0,1, \frac{1}{2} P \lambda_{s r}\right)\left(h_{0}\left(b_{r d}, a_{r d}, a_{s d}, b_{s d}\right)-h_{0}\left(a_{r d}, b_{r d}, a_{s d}, b_{s d}\right)\right) \\
&-\frac{a_{s d}}{b_{r d}+a_{s d}}\left(h_{4}\left(b_{r d}, a_{r d}, a_{s d}, b_{s d}, \frac{1}{2} P \lambda_{s r}\right)-h_{5}\left(b_{r d}, a_{r d}, a_{s d}, b_{s d}, \frac{1}{2} P \lambda_{s r}\right)\right) \\
&+\frac{b_{s d}}{a_{r d}+b_{s d}}\left(h_{4}\left(a_{r d}, b_{r d}, b_{s d}, a_{s d}, \frac{1}{2} P \lambda_{s r}\right)-h_{5}\left(a_{r d}, b_{r d}, b_{s d}, a_{s d}, \frac{1}{2} P \lambda_{s r}\right)\right) \\
&-\frac{a_{s d}}{a_{r d}+a_{s d}} h_{5}\left(a_{r d}, b_{r d}, a_{s d}, b_{s d}, \frac{1}{2} P \lambda_{s r}\right)+\frac{b_{s d}}{b_{r d}+b_{s d}} h_{5}\left(b_{r d}, a_{r d}, b_{s d}, a_{s d}, \frac{1}{2} P \lambda_{s r}\right) \\
& \stackrel{P \gg}{\approx} \frac{3}{4 P^{2} \lambda_{r d} \lambda_{s d}}+\frac{2}{P^{2} \lambda_{s r} \lambda_{s d}}=\mathrm{O}\left(\frac{1}{P^{2}}\right)
\end{aligned}
$$

$$
\begin{aligned}
& h_{4}(a, b, c, d, t)=\frac{b}{a+b} \frac{d}{c+d}\left(1-\sqrt{8(a+c)^{2} t} h_{3}\left(\sqrt{\frac{4(a+c)^{2} t}{\pi}}\right)\right) \\
& h_{5}(a, b, c, d, t)=\frac{b}{a+b} \frac{d}{c+d} \frac{2}{B \sqrt{t(2+t)}} \times\left(h_{3}\left(\sqrt{\frac{8(a+c)^{2} t}{\pi(2+t)}}\right)-h_{3}\left(\sqrt{\frac{t}{2(2+t)}}\left(\frac{4(a+c)}{\sqrt{\pi}}+A\right)\right)\right)
\end{aligned}
$$

channels $\left\{f_{1}, f_{2}\right\}$. At high SNRs, it can be approximated by

$$
P_{3} \stackrel{P}{\approx} \frac{4+2 \log \left(\frac{P \lambda_{s r}}{2}\right)}{P^{2} \lambda_{s r} \lambda_{s d}}+\frac{3}{P^{2} \lambda_{r d} \lambda_{s d}}=\mathrm{O}\left(\frac{\log P}{P^{2}}\right)
$$

By comparing (53b) and (61), we observe that Type-3 PEP is the dominant error term, thus the error rates of non-coherent DNC scale as $\mathrm{O}\left(\frac{\log P}{P^{2}}\right)$ at high SNRs.

Next we study the average Type-3 PEP of coherent DNC, which can be obtained after averaging (58) over the channel distributions of $f_{1}$ and $f_{2}$. Since the exact analysis is intractable, we shall make some approximations. As the first step, we approximate $P_{r, F}$ in (55) by

$$
P_{r, F} \approx Q\left(\sqrt{\frac{1}{2} P \lambda_{s r}|f|^{2}}\right) \approx h_{2}\left(\sqrt{\frac{1}{2} P \lambda_{s r}|f|^{2}}\right),
$$

where $|f|^{2}=2 \min \left(\left|f_{1}\right|^{2},\left|f_{2}\right|^{2}\right) \sim \exp (1)$, and

$$
h_{2}(x)=\frac{1}{B \sqrt{2 \pi} x}\left(1-\exp \left(-\frac{A}{\sqrt{2}} x\right)\right) \exp \left(-\frac{1}{2} x^{2}\right)
$$

for $A=1.98$ and $B=1.135$. The first approximation is quite tight from modest to high SNRs, as the relay detection performance is roughly determined by the worse source-relay channel. The advantage of such approximation is that the conditional PEP (58) now relies only on the single variable $|f|$. As for the second approximation, we use $Q(x) \approx h_{2}(x)$ [27]. Next, we observe that the average PEP depends largely on the small values of $|f|$, as the conditional PEP decreases very fast with the variable $|f|$ at high SNRs. As a result, we can approximate $T_{r, F}$ by

$$
T_{r, F}=\log \frac{1-P_{r, F}}{P_{r, F}} \stackrel{|f| \ll 1}{\approx} \sqrt{\frac{4}{\pi} P \lambda_{s r}|f|^{2}} .
$$

After plugging (62) and (64) back into (58) and doing some lengthy algebra, we can obtain (65) and (66), where $h_{4}(a, b, c, d, t)$ and $h_{5}(a, b, c, d, t)$ are respectively given by
TABLE II

SCALING LAWS OF THE ERROR RATES

\begin{tabular}{c|c|c}
\hline & ANC & DNC \\
\hline Coherent & $\mathrm{O}\left(\frac{\log P}{P^{2}}\right)$ & $\mathrm{O}\left(\frac{1}{P^{2}}\right)$ \\
Partial/Non-coherent & $\mathrm{O}\left(\frac{\log ^{3} P}{P^{2}}\right)$ & $\mathrm{O}\left(\frac{\log P}{P^{2}}\right)$ \\
\hline
\end{tabular}

(67) and (68), and $h_{3}(x)=\exp \left(\frac{x^{2}}{2}\right) Q(x)$. From (53a) and (66), we conclude that the error rates of coherent DNC scale as $\mathrm{O}\left(\frac{1}{P^{2}}\right)$ at high SNRs.

\section{Discussions}

We summarize the scaling laws of the error rates in Table II. It is observed that the error rates can be put in a general form of $P_{e}=\mathrm{O}\left(\frac{\log ^{d_{1}} P}{P^{d_{2}}}\right)$. The diversity order is defined as [1]

$$
\operatorname{div}=-\frac{\log P_{e}}{\log P}=d_{2}-d_{1} \frac{\log \log P}{\log P} .
$$

At extremely high SNRs (i.e., $P \rightarrow \infty$ ), the second term vanishes and the dominant diversity gain is $d_{2}$. But at modest SNRs, the logarithmic term would incur noticeable diversity loss and make the error curves decrease very slowly with SNRs. The extent of such diversity loss can be measured by $d_{1}$.

From Table II, we can observe that the dominant diversity gain is 2 regardless of the network coding scheme and the type of receiver. The diversity gain comes from node cooperation, as each source symbol can reach the destination through two independent paths, i.e., direct link and relay link. However, the logarithmic term loss is totally different for these schemes. For coherent detection, the logarithmic term loss of ANC has an order of 1 , whereas there is no logarithmic term loss for DNC. This is because for ANC, different source signals are randomly combined in the complex field through linear addition, so MUI would appear at the destination and degrade the performances of ML detection. On the contrary, for DNC the source signals 


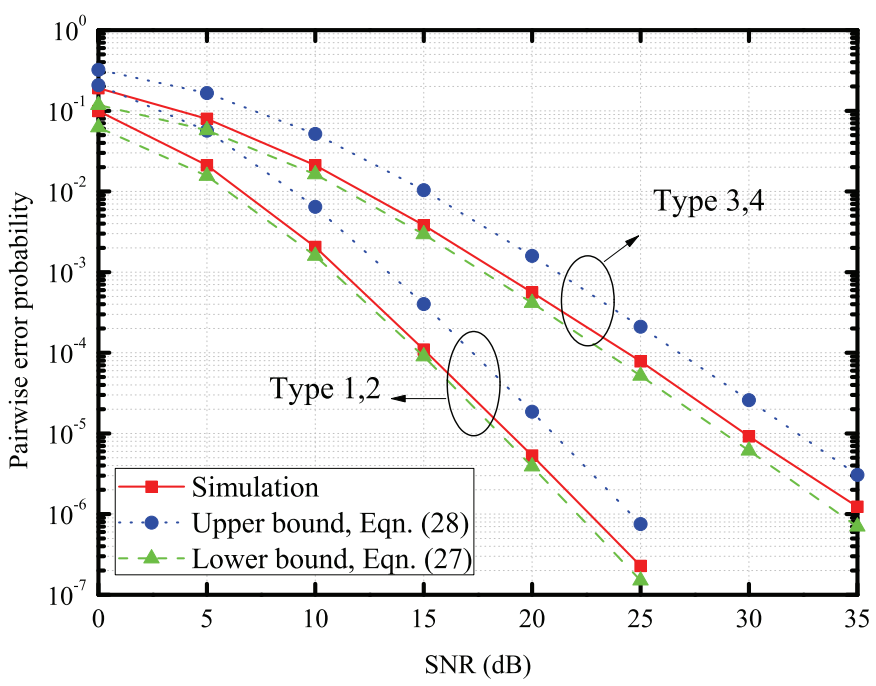

Fig. 2. PEP of coherent ANC.

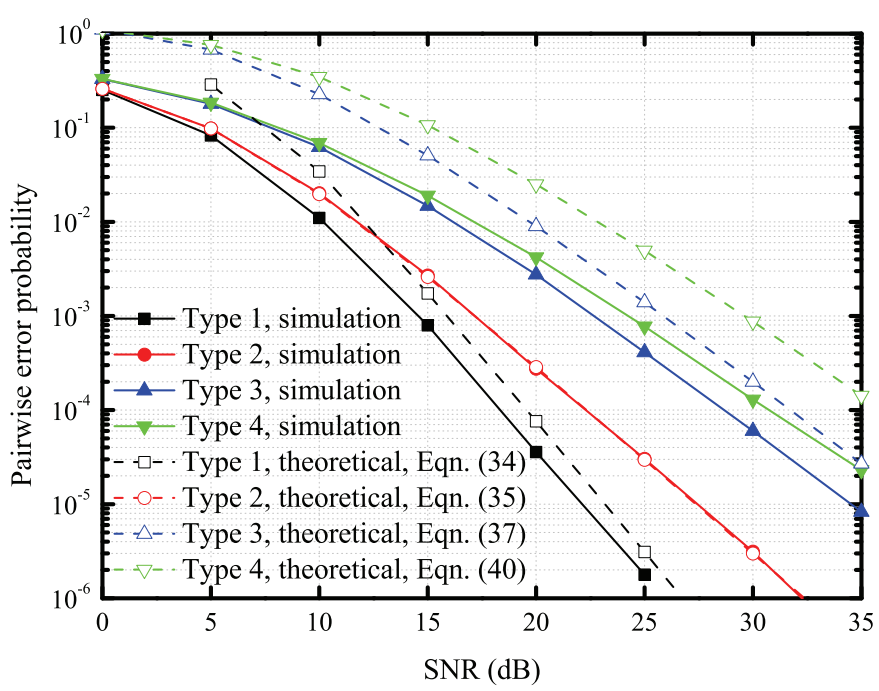

Fig. 3. PEP of partial coherent ANC.

are combined in the finite field through bit operation, thus the relay symbol is a single network-coded FSK symbol without any MUI.

For partial/non-coherent detection, the lack of perfect CSI would incur more logarithmic term loss for both ANC and DNC. Specifically, the order of the logarithmic term loss are 1 and 3 respectively for coherent ANC and partial coherent ANC, while for coherent DNC and non-coherent DNC the order of the logarithmic term loss are 0 and 1, respectively. As a result, the performance loss of ANC is more serious, and the reasons are given in the sequel. For DNC, the relay node may detect the wrong symbols and propagate these errors to the destination. So the relay link signal is properly scaled through nonlinear operations in the ML combiner according to the estimated relay detection error rates at the destination. For non-coherent detection, the real-time relay detection states are blind to the destination, and the scaling is based on the average relay detection error rates. Although such scaling is accurate on average, it cannot capture the instantaneous network dynamics and thus incurs some performance loss. The

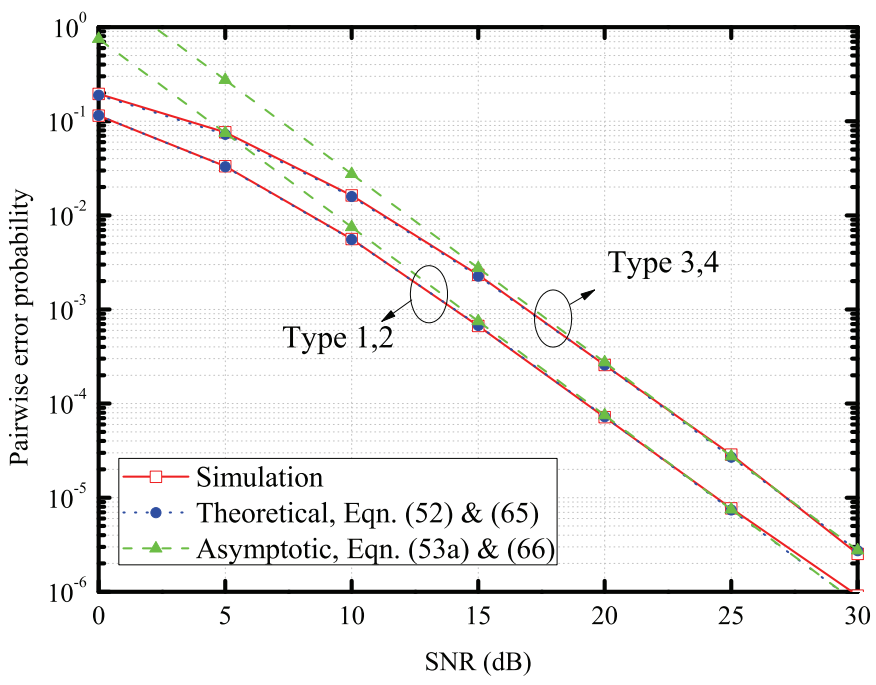

Fig. 4. PEP of coherent DNC.

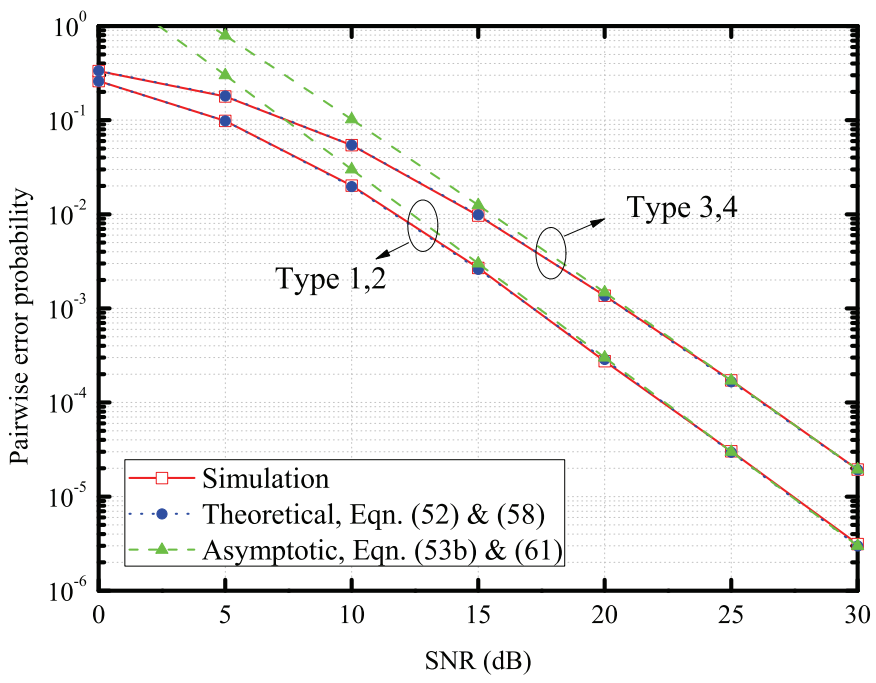

Fig. 5. PEP of non-coherent DNC.

situation becomes worse for ANC, as the formation of MUI is totally blind to the destination when perfect CSI is unknown. Consequently, the destination cannot efficiently suppress MUI to clearly separate the two source signals. That is why ANC suffers more serious performance loss than DNC when perfect CSI is unavailable at the destination.

Lastly, we would like to point out that the non-coherent detection may behave very differently for the single-user channels and the multi-user channels. For the single-user point-to-point channels, it is well known that non-coherent detection only brings 3dB SNR loss compared to coherent detection, but the diversity performances remain the same [24]. As for the multi-user network-coded uplink channels, we have demonstrated that non-coherent detection would incur diversity loss at modest SNRs, but the dominant diversity gains remain the same at extremely high SNRs.

\section{Simulations}

In this section, we present some simulation results to validate our analysis. The path loss model is $\lambda=D^{-3}$, where 


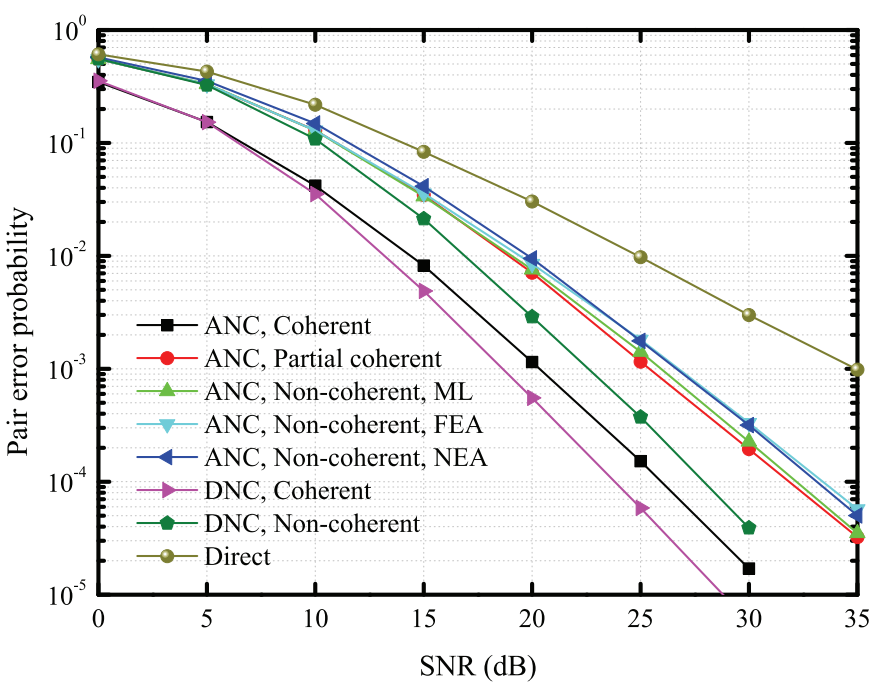

Fig. 6. Error rates of symmetric networks.

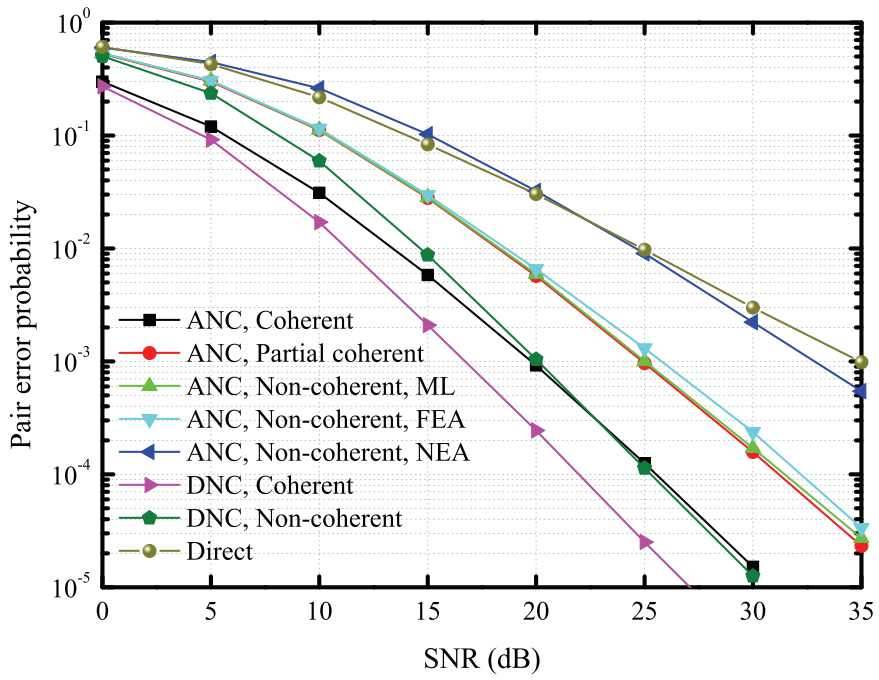

Fig. 7. Error rates of asymmetric networks with better source-relay channels.

$\lambda$ is the channel gain and $D$ is the distance between two terminals. Pair error probability is used as the performance metric, i.e., the probability that at least one of the source symbols is detected incorrectly at the destination. To simplify the simulation settings, only binary FSK modulation is studied and $D_{s d}$ is always normalized to 1 . In the simulations, direct transmission means the two source nodes take turns to deliver their own information to the destination without the help of the relay node. The transmitted power for direct transmission is normalized in such a way that the total energy consumption of each source node is identical to that for network coding.

First we compare the simulated PEPs with our theoretical results in the symmetric networks, where all the inter-node distances are normalized to 1 . For coherent ANC, we observe in Fig. 2 that the error curves are always between the two bounds. Besides, the two bounds have the same slopes at high SNRs, since their scaling laws are the same as shown in section IV.A. Next in Fig. 3, we plot the four types of PEPs of partial coherent ANC, where for Type-4 PEP only the upper bound is given. It is observed that all the error curves

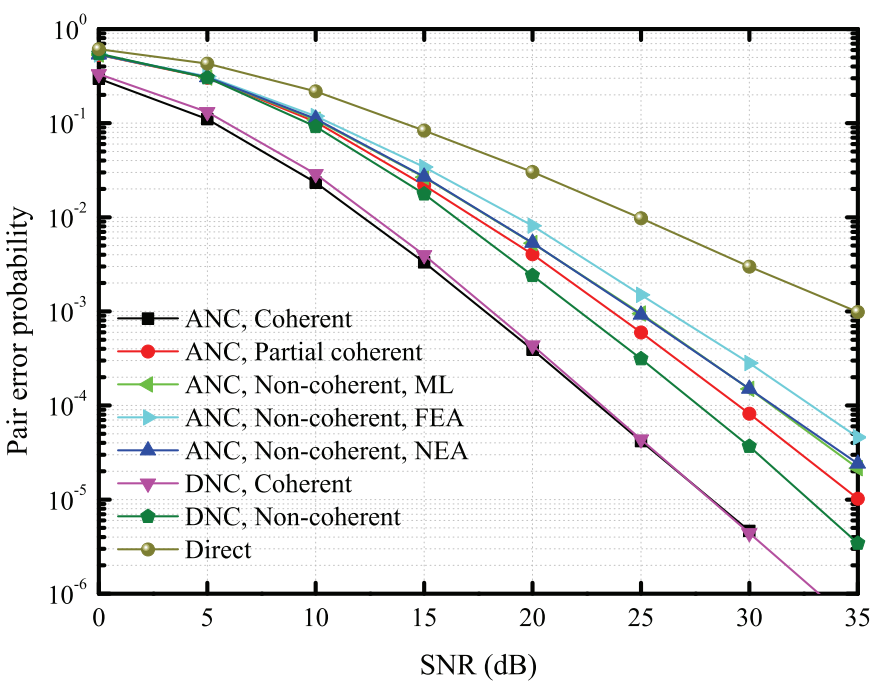

Fig. 8. Error rates of asymmetric networks with better relay-sink channels.

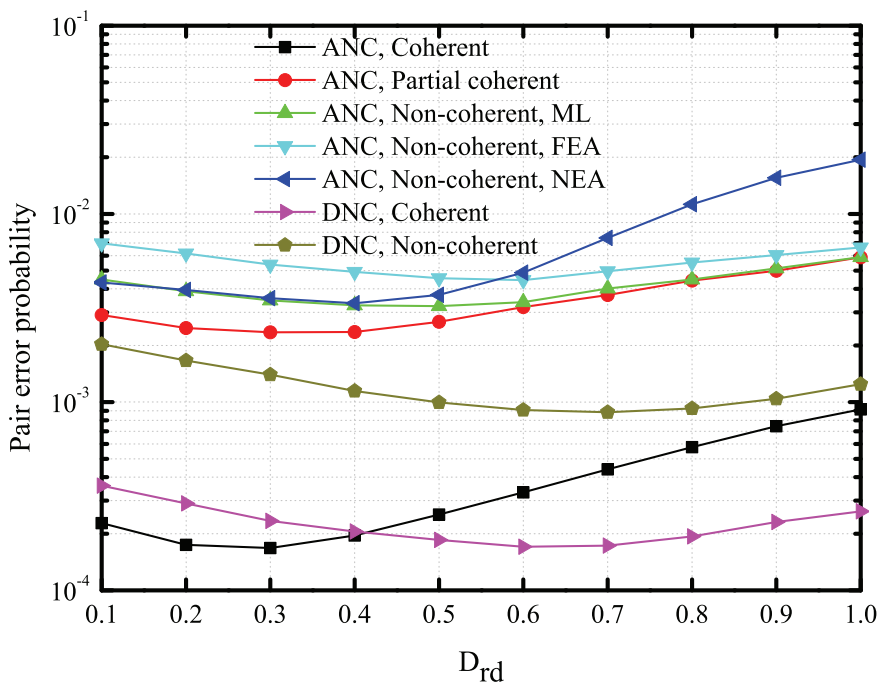

Fig. 9. Error rates with different relay positions.

have the same scaling behaviors as our theoretical results at high SNRs. Although Type-2, Type-3 and Type-4 PEPs have the same dominant diversity order of 2, the PEPs with larger logarithmic term loss decrease much more slowly at moderate SNRs. Then in Fig. 4 and Fig. 5, we show the PEPs of coherent and non-coherent DNC. It is observed that the two error curves of coherent DNC have the same slopes at high SNRs, whereas for non-coherent DNC Type-3 PEP suffers some diversity loss at modest SNRs. In all cases, our theoretical results match perfectly well with the simulation results.

Then in Fig. 6, we compare the real error rates in the symmetric networks. Compared to direct transmission without user cooperation, both coherent and non-coherent cooperation can provide a diversity order of 2 . For ANC, it is observed that the partial coherent receivers perform almost the same as non-coherent receivers. Besides, the two suboptimum noncoherent receivers also perform reasonably well with about $1 \mathrm{~dB}$ SNR loss compared to the ML receivers. Compared to coherent receivers, the partial/non-coherent receivers suffer huge performance loss due to the logarithmic term loss. For 
example, the non-coherent receivers have about 6dB SNR loss when the error rate is $10^{-4}$. For DNC, we can observe that the coherent receivers perform much better than the non-coherent receivers, but the performance gap is not as large as that of ANC. In all cases, DNC performs better than ANC using the same type of receivers. This is because the extent of the logarithmic term loss is smaller for DNC, and there is no MUI within the relay branch. Finally, we observe that the non-coherent DNC and coherent ANC have the same scaling behaviors at high SNRs, which is consistent with our analysis.

We also compare the error rates in the asymmetric networks. In Fig. 7, we study the networks with better source-relay channels, where $D_{s r}=0.4$ and all the other distances are normalized. By comparing Fig. 6 and Fig. 7, we observe that the performances of DNC have greatly improved, since better source-relay channel conditions would lead to reduced relay detection errors. On the contrary, the performances of ANC almost remain the same due to the power normalization effects at the relay node. For non-coherent $\mathrm{ANC}$, we observe that the FEA receivers perform very close to the ML receivers, whereas the NEA receivers suffer great performance loss, since neglecting the dominant noise terms on the second hop would greatly distort the likelihood function. Next in Fig. 8, we study the networks with better relay-destination channels, where $D_{r d}=0.4$ and all the other distances are normalized. We observe that improving the relay-destination channel conditions would bring huge coding gain to ANC. The performances of coherent ANC and DNC are almost indistinguishable, whereas for non-coherent receivers the performance gap is greatly reduced. For non-coherent ANC, the NEA receivers perform almost the same as the ML receivers, whereas the FEA receivers suffer about 1.5dB SNR loss.

Finally we study the error rates with different relay positions in Fig. 9, where the system SNR is $20 \mathrm{~dB}$. For the network topology, we place the destination at $(0,0)$, and locate the two source nodes at $\left(\frac{\sqrt{3}}{2}, \pm \frac{1}{2}\right)$. The relay node moves along the $\mathrm{x}$-axis from $(0.1,0)$ to $(1,0)$. The simulation results clearly show that the best performance of ANC is achieved when the relay node is close to the destination, whereas for DNC the best performance is achieved when the relay node is close to the sources. In all cases, non-coherent DNC performs better than the corresponding ANC due to the smaller logarithmic term loss. For non-coherent ANC, we observe that the NEA receivers are nearly optimum when the relay node is close to the destination, whereas the performances of the FEA receivers get closer to the performances of the ML receivers as the relay node moves to the two source nodes. Throughout simulations, we have observed that the partial coherent ANC performs very close to the non-coherent ANC. So we conjecture that these two schemes actually have the same scaling behaviors. A rigorous proof is deferred to our future work.

\section{CONCLUSIONS}

We study the two-user network-coded uplink where the receivers have non-perfect CSI. The transceivers for both ANC and DNC are developed under different CSI assumptions. It is demonstrated that non-coherent detection would incur diversity loss at modest SNRs, but the dominant diversity gains remain the same at extremely high SNRs. It is also demonstrated that the non-coherent ANC would incur more serious performance loss than the non-coherent DNC as the destination cannot efficiently suppress MUI.

\section{REFERENCES}

[1] K. J. Ray Liu, A. K. Sadek, W. Su, and A. Kwasinski, Cooperative Communications and Networking. Cambridge University Press, 2008.

[2] J. N. Laneman, D. N. C. Tse, and G. W. Wornell, "Cooperative diversity in wireless networks: efficient protocols and outage behavior," IEEE Trans. Inf. Theory, vol. 50, no. 12, pp. 3062-3080, Dec. 2004.

[3] R. Ahlswede, N. Cai, S.-Y. R. Li, and R. W. Yeung, "Network information flow," IEEE Trans. Inf. Theory, vol. 46, no. 4, pp. 1204-1216, July 2000

[4] S. Zhang, S. C. Liew, and P. P. Lam, "Physical-layer network coding," in Proc. 2006 ACM MOBICOM.

[5] W. Guan and K. J. Ray Liu, "Performance analysis of two-way relaying with non-coherent differential modulation," IEEE Trans. Wireless Commun., vol. 10, no. 6, pp. 2004-2014, June 2011.

[6] S. Katti, S. Gollakota, and D. Katabi, "Embracing wireless interference: analog network coding," in Proc. 2007 ACM SIGCOMM.

[7] W. Guan and K. J. Ray Liu, "Mitigating error propagation for wireless network coding," IEEE Trans. Wireless Commun., vol. 11, no. 10, pp. 3632-3643, Oct. 2012.

[8] W. Guan and K. J. Ray Liu, "Diversity analysis of analog network coding with multi-user interferences," to appear in IEEE Trans. Wireless Commun., 2013.

[9] W. Guan and K. J. Ray Liu, "Error performances of multiple access system using analog network coding," in Proc. 2012 IEEE Int. Conf. Commun.

[10] D. Chen and J. N. Laneman, "Cooperative diversity for wireless fading channels without channel state information," in Proc. 2004 Asilomar Conf. Signals, Syst. Comput., pp. 1307-1312.

[11] R. Annavajjala, P. C. Cosman, and L. B. Milstein, "On the performance of optimum noncoherent amplify-and-forward reception for cooperative diversity," in Proc. 2005 IEEE Military Commun. Conf., pp. 3280-3288.

[12] Y. Zhu, P. Y. Kam, and Y. Xin, "Non-coherent detection for amplifyand-forward relay systems in a Rayleigh fading environment," in Proc. 2007 IEEE Global Telecommun. Conf., pp. 1658-1662.

[13] G. Farhadi and N. C. Beaulieu, "A low complexity receiver for noncoherent amplify-and-forward cooperative systems," IEEE Trans. Wireless Commun., vol. 58, no. 9, pp. 2499-2504, Sept. 2010.

[14] M. R. Souryal, "Non-coherent amplify-and-forward generalized likelihood ratio test receiver,' IEEE Trans. Wireless Commun., vol. 9, no. 7, pp. 2320-2327, July 2010.

[15] D. Chen and J. N. Laneman, "Modulation and demodulation for cooperative diversity in wireless systems," IEEE Trans. Wireless Commun., vol. 5, no. 7, pp. 1785-1794, July 2006.

[16] T. Himsoon, W. P. Siriwongpairat, W. F. Su, and K. J. Ray Liu, "Differential modulation with threshold-based decision combining for cooperative communications," IEEE Trans. Signal Process., vol. 55, no. 7, pp. 3905-3923, July 2007.

[17] J. H. Yuan, Y. H. Li, and L. Chu, "Differential modulation and relay selection with detect-and-forward cooperative relaying," IEEE Trans. Veh. Technol., vol. 59, no. 1, pp. 261-268, Jan. 2010.

[18] T. Cui, F. F. Gao, and C. Tellambura, "Differential modulation for two-way wireless communications: a perspective of differential network coding at the physical layer," IEEE Trans. Commun., vol. 57, no. 10, pp. 2977-2987, Oct. 2009.

[19] L. Y. Song, G. Hong, B. L. Jiao, and M. Debbah, "Joint relay selection and analog network coding using differential modulation in two-way relay channels," IEEE Trans. Veh. Technol., vol. 59, no. 6, pp. 29322939, July 2010.

[20] I. Krikidis, Z. G. Ding, and C. D. Charalambous, "Noncoherent energy detection with orthogonal signaling for an uncoded two-way relay channel," IEEE Trans. Veh. Technol., vol. 61, no. 1, pp. 404-409, Jan. 2012.

[21] M. C. Valenti, D. Torrieri, and T. Ferrett, "Noncoherent physical-layer network coding with FSK modulation: relay receiver design issues," IEEE Trans. Commun., vol. 59, no. 9, pp. 2595-2604, Sept. 2011.

[22] J. Tian, Q. Zhang, and F. Q. Yu, "Non-coherent detection for two-way AF cooperative communications in fast Rayleigh fading channels," IEEE Trans. Commun., vol. 59, no. 10, pp. 2753-2762, Oct. 2011. 
[23] M. Abramovitz and I. A. Stegun, Handbook of Mathematical Functions with Formulas, Graphs, and Mathematical Tables, 9th edition. Dover, 1972.

[24] J. Proakis, Digital Communications, 4th edition. McGraw-Hill, 2001.

[25] I. S. Gradshteyn and I. M. Ryzhik, Table of Integrals, Series, and Products, 7th edition. Academic, 2007.

[26] K. H. Biyari and W. C. Lindsey, "Statistical distributions of hermitian quadratic forms in complex Gaussian variables," IEEE Trans. Inf. Theory, vol. 39, no. 3, pp. 1076-1082, May 1993.

[27] G. K. Karagiannidis and A. S. Lioumpas, "An improved approximation for the Gaussian Q-function," IEEE Commun. Lett., vol. 11, no. 8, pp. 644-646, Aug. 2007.

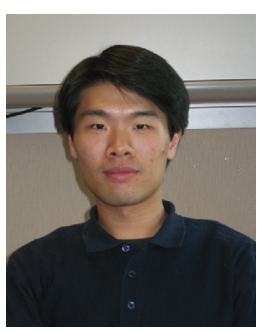

Wei Guan (S'12) received the B.S. in Electrical Engineering and Finance (double degree) in 2006, and M.S. (with highest honor) in Electrical Engineering in 2009, both from Shanghai JiaoTong University, Shanghai, China. Now he is a Ph.D. student in the Department of Electrical and Computer Engineering at University of Maryland, College Park.

His current research interests are in the areas of wireless communications and networks, including cooperative communications and network coding. $\mathrm{He}$ received the 1st Prize in the 18th National Physics Contest, Shanghai, and the A. James Clark School of Engineering Distinguished Graduate Fellowship from University of Maryland, College Park in 2009.

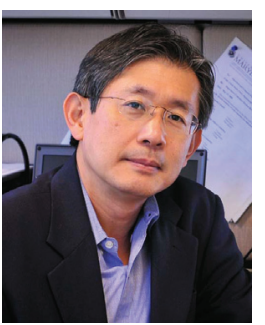

K. J. Ray Liu (F'03) was named a Distinguished Scholar-Teacher of University of Maryland, College Park, in 2007, where he is Christine Kim Eminent Professor of Information Technology. He leads the Maryland Signals and Information Group conducting research encompassing broad areas of signal processing and communications with recent focus on cooperative communications, cognitive networking, social learning and networks, and information forensics and security.

Dr. Liu is the recipient of numerous honors and awards including IEEE Signal Processing Society Technical Achievement Award and Distinguished Lecturer. $\mathrm{He}$ also received various teaching and research recognitions from University of Maryland including university-level Invention of the Year Award; and Poole and Kent Senior Faculty Teaching Award and Outstanding Faculty Research Award, both from A. James Clark School of Engineering. An ISI Highly Cited Author in Computer Science, Dr. Liu is a Fellow of IEEE and AAAS.

Dr. Liu is President of IEEE Signal Processing Society where he has served as Vice President - Publications and Board of Governor. He was the Editor-inChief of IEEE Signal Processing Magazine and the founding Editor-in-Chief of EURASIP Journal on Advances in Signal Processing. 